
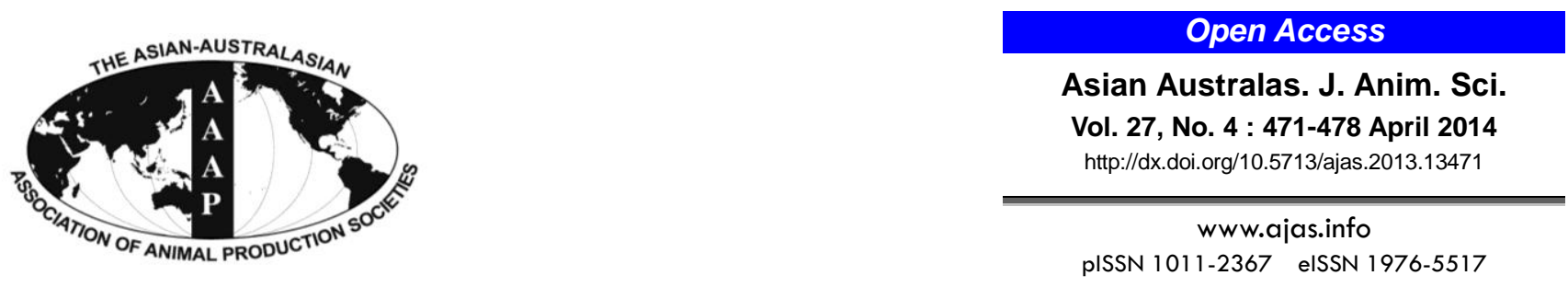

\title{
Selection of Reference Genes for Gene Expression Studies in Porcine Whole Blood and Peripheral Blood Mononuclear Cells under Polyinosinic:Polycytidylic Acid Stimulation
}

\author{
Jiying Wang, Yanping Wang, Huaizhong Wang, Xiaojing Hao ${ }^{1}$, Ying Wu*, and Jianfeng Guo* \\ Shandong Provincial Key Laboratory of Animal Disease Control and Breeding, \\ Institute of Animal Science and Veterinary Medicine, \\ Shandong Academy of Agricultural Sciences, Jinan 250100, China
}

\begin{abstract}
Investigating gene expression of immune cells of whole blood or peripheral blood mononuclear cells (PBMC) under polyinosinic:polycytidylic acid (poly I:C) stimulation is valuable for understanding the immune response of organism to RNA viruses. Quantitative real-time PCR (qRT-PCR) is a standard method for quantification of gene expression studies. However, the reliability of qRT-PCR data critically depends on proper selection of reference genes. In the study, using two different analysis programs, geNorm and NormFinder, we systematically evaluated the gene expression stability of six candidate reference genes $(G A P D H, A C T B, B 2 M, R P L 4$, $T B P$, and PPIA) in samples of whole blood and PBMC with or without poly I:C stimulation. Generally, the six candidate genes performed a similar trend of expression stability in the samples of whole blood and PBMC, but more stably expressed in whole blood than in PBMC. geNorm ranked B2M and PPIA as the best combination for gene expression normalization, while according to NormFinder, $T B P$ was ranked as the most stable reference gene, followed by $B 2 M$ and PPIA. Comprehensively considering the results from the two programs, we recommended using the geometric mean of the three genes, $T B P, P P I A$ and $B 2 M$, to normalize the gene expression of whole blood and PBMC with poly I:C stimulation. Our study is the first detailed survey of the gene expression stability in whole blood and PBMC with or without poly I:C stimulation and should be helpful for investigating the molecular mechanism involved in porcine whole blood and PBMC in response to poly I:C stimulation. (Key Words: Pigs, Reference Genes, Whole Blood, Peripheral Blood Mononuclear Cells (PBMC), Polyinosinic:Polycytidylic Acid (Poly I:C))
\end{abstract}

\section{INTRODUCTION}

Quantitative real-time PCR (qRT-PCR) can simultaneously measure gene expression in many different samples for a limited number of genes, and is especially suitable when only a small number of cells are available. It has become the standard method for quantification of gene expression studies. In case of qRT-PCR, the use of reference

\footnotetext{
* Corresponding Authors: Ying Wu. Tel: +86-0531-88613698, Fax: +86-0531-88613698, E-mail: wusaas@163.com / Jianfeng Guo. Tel: +86-0531-88978796, Fax: +86-0531-8897 8476, E-mail: Guojf982@163.com

${ }^{1}$ Qingdao Institute of Animal Science and Veterinary Medicine, Qingdao 266100, China.

Submitted Aug. 5, 2013; Accepted Nov. 20, 2013; Revised Dec. 16, 2013
}

genes as internal controls is the most common method for normalizing mRNA data (Huggett et al., 2005). The reference gene is a stably expressed gene that is experimentally verified in given species and tissues under given experimental conditions. However, an increasing number of reports have shown that the expression of some frequently used internal reference genes may vary significantly under different experimental conditions (Vandesompele et al., 2002; Nygard et al., 2007; Facci et al., 2011). If the chosen reference gene has a large expression fluctuation among samples tested, the normalization will lead to erroneous gene expression profiles of the target gene of interest. Therefore, the selection of the most stable gene or set of genes as internal controls is a critical step to control the variability among samples for quantitative gene 
expression studies with a sensitive qRT-PCR technique.

Whole blood, especially peripheral blood mononuclear cells (PBMC) isolated from it, consists of many types of immune cells, such as lymphocytes, granulocyte and monocytes/macrophages, can produce different receptors and cytokines that lead to immediate innate immune responses (Gao et al., 2010; Uddin et al., 2012). As in vitro models, they have been widely used to dissect the pathogenesis and genetics behind infection and stimulation through gene expression studies (Gao et al., 2010; Uddin et al., 2012). Polyinosinic:polycytidylic acid (poly I:C) is an analogue of viral double-stranded RNA (dsRNA) that can serve as pathogen-associated molecular pattern and activates innate immunity through two major dsRNA sensors, toll-like receptor 3 (TLR3) and melanoma differentiation associated protein-5 (MDA5) (McCartney et al., 2009). Using the poly I:C as an immunologic stimulant is an important experimental measure to study the immune regulation of an organism to RNA viruses (Caskey et al., 2011; Cao et al., 2013).

Pigs are not only one of the most economically important livestock worldwide, but an important research animal that has become a useful model for various human diseases (Meurens et al., 2012). However, no studies have been performed to validate the stability of reference genes in samples of porcine whole blood and PBMC in response to poly I:C stimulation. Therefore, the aim of the current study was to identify a set of stably expressed reference genes in porcine whole blood and PBMC in response to poly I:C stimulation. The reference genes selected in the study would be helpful for investigating the molecular mechanisms involved in porcine blood and PBMC in response to poly I:C stimulation.

\section{MATERIAL AND METHODS}

\section{Blood collection and PBMC isolation}

Three clinically healthy Landrace pigs of 35 days old were bled to obtain blood samples. For each piglet, $20 \mathrm{~mL}$ blood was collected via venipuncture into a vacutainer tube containing anticoagulant (EDTAK2). Out of the $20 \mathrm{~mL}$ whole blood per piglet, $15 \mathrm{~mL}$ was used for isolation of PBMC while the other $5 \mathrm{~mL}$ for whole blood culture. The whole study protocols for collection of the blood from experimental pigs were reviewed and approved by the Institutional Animal Care and Use Committee (IACUC) of Shandong Academy of Agricultural Sciences.

PBMC were isolated using Ficoll-Hypaque PLUS (GE healthcare) following the manufacturer's instructions. In brief, the whole blood was firstly diluted by an equal volume of balanced salt solution (PBS). Then, $15 \mathrm{~mL}$ of diluted blood was carefully added on the top of $10 \mathrm{~mL}$ of Ficoll-Hypaque solution in a $50 \mathrm{~mL}$ conical tube and centrifuged at $460 \times \mathrm{g}$ for $20 \mathrm{~min}$ at room temperature. After centrifugation, the middle whitish interface containing mononuclear cells was transferred to a new tube, and washed by adding $25 \mathrm{~mL}$ PBS followed by centrifugation at $150 \times \mathrm{g}$ for $10 \mathrm{~min}$ two times.

\section{Poly I:C stimulation}

PBMC, isolated from $15 \mathrm{~mL}$ blood of every pig, were diluted into $50 \mathrm{~mL}$ RPMI-1640 medium (Hyclone) supplemented with $10 \%$ fetal calf serum, streptomycin and penicillin, with the final cell concentration of $\sim 2 \times 10^{6} / \mathrm{mL}$. The cell suspension was divided into two parts, one of $10 \mathrm{ml}$, used as the uncultured and un-stimulated control, the other $40 \mathrm{~mL}$, which was equally assigned into 16 wells (2.5 $\mathrm{mL} /$ well) for the stimulation test. Then, these 16 wells were divided into four time-point groups, which were cultured for $3 \mathrm{~h}, 6 \mathrm{~h}, 12 \mathrm{~h}$, and $24 \mathrm{~h}$ at $37^{\circ} \mathrm{C}$ with $5 \% \mathrm{CO}_{2}$. Additionally, the four wells of every group were used for stimulation with different poly I:C concentration $(0,10,20$, $40 \mu \mathrm{g} / \mathrm{mL}$, respectively) (Sigma-Aldrich).

In order to assure a sufficient amount of cells for the next RNA extraction, samples of the three piglets with the same concentration of poly I:C and stimulation time were merged together for RNA extraction. Thus, we had 17 samples of PBMC, including five samples without poly I:C stimulation (one uncultured control and 4 samples collected after $3 \mathrm{~h}, 6 \mathrm{~h}, 12 \mathrm{~h}$, and $24 \mathrm{~h}$ of culture) and 12 samples with poly I:C stimulation (4 time points, and each point with 3 different poly I:C concentration tests).

The other $5 \mathrm{~mL}$ fresh blood was diluted by adding 20 mL RPMI-1640 medium supplemented with $10 \%$ fetal calf serum, streptomycin and penicillin. About $20 \mathrm{~mL}$ diluted whole blood was equally assigned into 16 wells (1.25 $\mathrm{mL} /$ well) and the other $5 \mathrm{~mL}$ were used as the uncultured and un-stimulated control. According to the sample experimental treatments as PBMC, the diluted whole blood were stimulated and collected, and samples of the three pigs were merged.

\section{RNA extraction, cDNA synthesis and quantitative real- time PCR}

Total RNA was isolated from samples of whole blood and PBMC using the TRIzol reagent according to the manufacturer's protocol (Invitrogen, Burlington, Canada). The quantity and quality of RNA was determined by gel electrophoresis and NanoDrop 2000 spectrophotometer (Thermo Scientific). The PrimeScript RT reagent kit with gDNA Eraser (RR047A, Takara, Japan) was used to remove possible contaminating genomic DNA. The extracted RNA was firstly treated with gDNA Eraser for $2 \mathrm{~min}$ at $42^{\circ} \mathrm{C}$, and then reverse-transcribed into cDNA for the mRNA reference gene analysis according to the instructions of the manufacturer. The final cDNA products were diluted 4 -fold 
prior to use in qRT-PCR.

Six candidate reference genes, namely glyceraldehyde 3-phosphate dehydrogenase $(G A P D H)$, beta-actin $(A C T B)$, beta-2-microglobulin $(B 2 M)$, ribosomal protein L4 (RPL4), TATA-box binding protein $(T B P)$ and cyclophilin A (PPIA), were selected from the most commonly used ones reported in previous studies (Martino et al., 2011; Cinar et al., 2012). The function, primers and other related information of these candidate reference genes are listed in Table 1.

All qRT-PCR were carried out using LightCycler 480 SYBR Green I Master on Roche LightCycler 480 instrument following the manufacturer's guidelines. Prior to performing the expression stability assay for all the primers of the six candidate genes, we carried out melting curve analyses to detect their specificity, and then calculated the PCR amplification efficiencies of every primer using a standard curve derived from a pooled cDNA mixture serially diluted 4-fold over five measuring points. In the expression stability assay, all the samples were amplified in duplicate. The PCR reaction consisted of $10 \mu \mathrm{L}$ BlueSYBR-Green mix, $1 \mu \mathrm{L}$ forward and reverse primers (10 $\mathrm{pM} / \mu \mathrm{L}), 7 \mu \mathrm{L}$ distilled water, and $1 \mu \mathrm{L}$ of cDNA in a total volume of $20 \mu \mathrm{L}$. The thermal cycling conditions were 3 min at $95^{\circ} \mathrm{C}$, followed by 40 reaction cycles $\left(10 \mathrm{~s}\right.$ at $95^{\circ} \mathrm{C}$ and $20 \mathrm{~s}$ at $60^{\circ} \mathrm{C}$ ). The second derivative maximum algorithm included within the instrument software was used to determine cycle threshold $(\mathrm{Ct})$ values for each reaction.

\section{Analyses of reference gene expression stability}

The data obtained from the qRT-PCR assays were converted into correct input files, according to the requirements of the software, and analyzed using geNorm version 3.5 (Vandesompele et al., 2002) and NormFinder (version 0.953) (Andersen et al., 2004). In brief, geNorm calculates the gene expression stability measure $\mathrm{M}$ for a reference gene as the average pairwise variation $\mathrm{V}$ for that gene with all other tested reference genes. Besides the $M$ values, geNorm also calculates average expression stability values of remaining control genes during stepwise exclusion of the least stable control gene. Finally, to determine the optimal number of control genes for normalization, by the program, we calculated the pairwise variation $\mathrm{V}_{\mathrm{n}} / \mathrm{V}_{\mathrm{n}+1}$ between two sequential normalization factors $\mathrm{NF}_{\mathrm{n}}$ and $\mathrm{NF}_{\mathrm{n}+1}$ containing an increasing number of reference genes. NormFinder uses the model-based strategy to estimate not only the overall expression variation of the candidate normalization genes, but also the variation between sample subgroups of the sample set, i.e. nonstimulation group and stimulation group in the study. The output of the program provides a stability value for each gene, which is a direct measure for the estimated expression variation enabling the user to evaluate the systematic error introduced when using the gene for normalization.

Student's t-test analysis for unpaired samples was also performed to evaluate statistical differences between samples with and without poly I:C stimulation.

\section{RESULTS}

\section{RNA quality and concentration of the PBMC and whole blood samples}

Total RNA of the samples of PBMC and whole blood was extracted using the TRIzol reagent, and their quality was measured by spectrophotometer. For the RNA of PBMC samples, the ratio A260/A280 was $1.94 \pm 0.25$ (average $\pm \mathrm{SD}$ ), and the concentration $78.2 \mathrm{ng} / \mu \mathrm{L} \pm 48.5$ (average $\pm \mathrm{SD}$ ). For the RNA of whole blood samples, the ratio A260/A280 was 2.00 \pm 0.06 (average $\pm S D$ ), and the concentration $273.9 \mathrm{ng} / \mu \mathrm{L} \pm 93.6$ (average $\pm \mathrm{SD}$ ). Our results indicated that all the RNA samples were good in quality and suitable for the next analysis. However, the concentration of RNA from PMBC was quite low, and larger volume samples should be used in the next cDNA synthesis.

\section{Verification of the primers for the candidate reference genes}

Using the mix pool RNA of the PBMC and whole blood samples, we generated amplification efficiency and melting curve analyses for the primers of the six candidate reference genes, and their results were shown in the last column of

Table 1. Detail information of selected candidate reference genes for mRNA expression

\begin{tabular}{|c|c|c|c|c|c|c|c|}
\hline Gene & Full name & Function & $\begin{array}{c}\text { GenBank } \\
\text { accession } \\
\text { number }\end{array}$ & Primer (5'-3') & Reference & $\begin{array}{l}\text { Length } \\
\text { (bp) }\end{array}$ & $\begin{array}{c}\text { qPCR } \\
\text { efficiency }\end{array}$ \\
\hline$\overline{G A P D H}$ & $\begin{array}{l}\text { Glyceraldehyde-3-phosphate } \\
\text { dehydrogenase }\end{array}$ & Carbohydrate metabolism & AF017079 & $\begin{array}{l}\text { F: TCGGAGTGAACGGATTTG } \\
\text { R: CCTGGAAGATGGTGATGG }\end{array}$ & $\begin{array}{l}\text { Martino et al., } \\
2011\end{array}$ & 219 & 97.90 \\
\hline$A C T B$ & Beta-actin & $\begin{array}{l}\text { Involved in cell motility, structure } \\
\text { and integrity }\end{array}$ & DQ178130 & $\begin{array}{l}\text { F: TCTGGCACCACACCTTCT } \\
\text { R: GATCTGGGTCATCTTCTCAC }\end{array}$ & $\begin{array}{l}\text { Martino et al., } \\
2011\end{array}$ & 114 & 101.20 \\
\hline$B 2 M$ & Beta-2-microglobulin & $\begin{array}{l}\text { Ctoskeletal protein involved } \\
\text { in cell locomotion }\end{array}$ & NM 213978 & $\begin{array}{l}\text { F: TTCACACCGCTCCAGTAG } \\
\text { R:CCAGATACATAGCAGTTCAGG }\end{array}$ & $\begin{array}{l}\text { Martino et al., } \\
2011\end{array}$ & 166 & 109.30 \\
\hline$T B P$ & TATA binding protein & $\begin{array}{l}\text { Transcription initiation } \\
\text { from RNA polymerase II promotor }\end{array}$ & DQ178129 & $\begin{array}{l}\text { F: GATGGACGTTCGGTTTAGG } \\
\text { R: AGCAGCACAGTACGAGCAA }\end{array}$ & $\begin{array}{l}\text { Martino et al., } \\
2011\end{array}$ & 124 & 101.40 \\
\hline RPLA & Ribosomal protein L4 & Structural constituent of ribosome & DQ845176 & $\begin{array}{l}\text { F: AGGAGGCTGTTCTGCTTCTG } \\
\text { R: TCCAGGGATGTTTCTGAAGG }\end{array}$ & $\begin{array}{l}\text { Cinar et al., } \\
2012\end{array}$ & 185 & 97.8 \\
\hline PPIA & $\begin{array}{l}\text { Peptidylprolyl isomerase A } \\
\text { (cyclophilin A) }\end{array}$ & $\begin{array}{l}\text { A member of the peptidyl-prolyl } \\
\text { cis-trans isomerase (PPIase) family }\end{array}$ & NM_214353 & $\begin{array}{l}\text { F: CACAAACGGTTCCCAGTTTT } \\
\text { R: TGTCCACAGTCAGCAATGGT }\end{array}$ & $\begin{array}{l}\text { Cinar et al., } \\
2012\end{array}$ & 171 & 95.3 \\
\hline
\end{tabular}


Table 2. Expression levels of candidate reference genes for mRNA expression analysis with or without poly I: C stimulation

\begin{tabular}{llcccccc}
\hline Sample type & Experimental group & GAPDH & $A C T B$ & $B 2 M$ & $T B P$ & $R P L 4$ & $P P I A$ \\
\hline PBMC $^{1}$ & Non-stimulation & $30.18 \pm 1.72$ & $20.75 \pm 1.00^{\mathrm{a}}$ & $22.24 \pm 1.22$ & $29.35 \pm 1.23$ & $25.73 \pm 1.83$ & $22.28 \pm 1.03$ \\
& Poly I:C stimulation & $30.55 \pm 1.74$ & $22.15 \pm 1.29^{\mathrm{b}}$ & $22.07 \pm 1.18$ & $29.38 \pm 1.15$ & $25.14 \pm 1.62$ & $22.33 \pm 1.00$ \\
& All samples of PBMC & $30.42 \pm 1.68$ & $21.68 \pm 1.36$ & $22.13 \pm 1.16$ & $29.37 \pm 1.14$ & $25.34 \pm 0.66$ & $22.32 \pm 0.98$ \\
Whole blood & Non-stimulation & $31.55 \pm 1.11$ & $22.07 \pm 1.46$ & $24.27 \pm 1.48$ & $31.38 \pm 1.05$ & $28.66 \pm 1.67$ & $24.56 \pm 1.66$ \\
& Poly I: C stimulation & $30.95 \pm 1.64$ & $22.48 \pm 0.85$ & $23.53 \pm 1.34$ & $31.42 \pm 1.48$ & $28.83 \pm 1.97$ & $24.10 \pm 1.46$ \\
& All samples of & $31.15 \pm 1.48$ & $22.34 \pm 1.06$ & $23.78 \pm 1.39$ & $31.41 \pm 1.32$ & $28.77 \pm 1.82$ & $24.26 \pm 1.50$ \\
& whole blood & & & & & & \\
All samples & & $30.79 \pm 1.60$ & $22.01 \pm 1.25$ & $22.95 \pm 1.52$ & $30.39 \pm 1.60$ & $27.05 \pm 2.45$ & $23.29 \pm 1.59$ \\
\hline
\end{tabular}

Expression levels of candidate reference genes were denoted by average \pm standard deviation (SD).

Gene with different superscript indicates significant difference between gene expression levels in poly I:C stimulation group and non-stimulation group by t-test $(\mathrm{p} \leq 0.05)$.

${ }^{1}$ PBMC $=$ Peripheral blood mononuclear cells.

Table 1 and supplementary Figure S1 (See e-version for supplement.), respectively. The amplification efficiencies for the six candidate reference genes ranged between $95.3 \%$ and $109.30 \%$. The melting curves of the six pairs of primers were single, smooth and sharply defined with a narrow peak, suggesting all the six pairs of primers produce pure and homogeneous products. Thus, all the primers for the six candidate reference gene were specific and suitable for further qRT-PCR assays.

\section{Expression profiles of candidate reference genes}

The cycle threshold $(\mathrm{Ct})$ values for the six $(G A P D H$, $A C T B, B 2 M, T B P, R P L 4$, and PPIA) candidate reference genes are listed in Table 2. The $\mathrm{Ct}$ values for the six genes studied ranged from $20.75(A C T B)$ to $31.55(G A P D H)$. Compared between samples of whole blood and PBMC, the 6 mRNA had similar expression trends. Out of the 6 mRNA candidate reference genes, ACTB ( $\mathrm{Ct} 22.01), B 2 M(\mathrm{Ct}$ 22.95) and PPIA (Ct 23.29) had high expression levels, followed by RPL4 (Ct 25.34), TBP (Ct 29.37), and GAPDH (Ct 30.42) with the lowest level. When expression values were compared between samples with or without Poly I:C stimulation, only $A C T B$ was expressed differentially ( $\mathrm{p}<$ 0.05) in PBMC samples, while no significant difference was observed for the other genes in the whole blood as well as in PBMC samples.

\section{Gene expression stability assessment using geNorm}

Gene expression stability measure $\mathrm{M}$ was calculated for the samples of whole blood and PBMC with or without poly I:C stimulation. Genes with the lowest $M$ value have the most stable expression, while the highest $M$ value has the least stable expression. The expression $M$ values of the six reference genes are plotted in Figure 1. It can be seen that, except more stably expressed in samples of whole blood than those of PBMC, the six candidate reference genes perform similar trend of expression stability in the sample of whole blood and PBMC. That is, B2M is the most stable reference gene with the lowest $\mathrm{M}$ value $(0.631$ and 0.777 in whole blood and PBMC, respectively), while $A C T B$ the least stable one with the highest $\mathrm{M}$ value $(0.921$ and 1.426 in whole blood and PBMC, respectively).

Assuming that the reference genes are not co-regulated, stepwise exclusion of the gene with the highest $M$ value results in a combination of two constitutively expressed reference genes that have the most stable expression in the tested samples (Vandesompele et al., 2002). Figure 2a and $2 b$ shows the average expression stability values of remaining control genes for whole blood and PBMC. Combining using the two reference genes for normalization, $B 2 M$ and PPIA, the average expression stability values can obtain rather small $\mathrm{M}$ values for the samples of whole blood (0.299) and PBMC (0.490).

In addition to a ranking regarding the expression stability of each gene, geNorm also calculates a normalization factor for each sample based on the most stable reference genes, which are illustrated in Figure 3. A large variation means that the added gene has a significant effect on the normalization and should preferably be included for calculation of a reliable normalization factor.

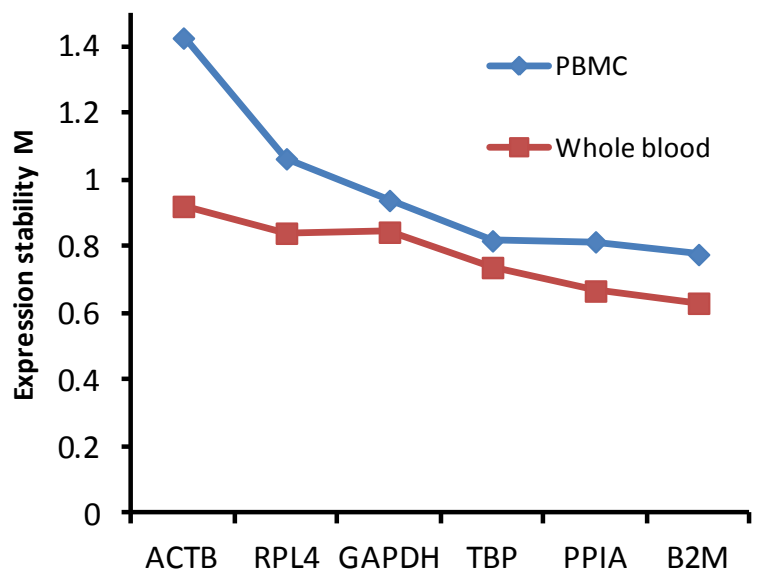

Figure 1. Gene expression stability ( $M$ values) of the six candidate reference genes calculated by geNorm. 


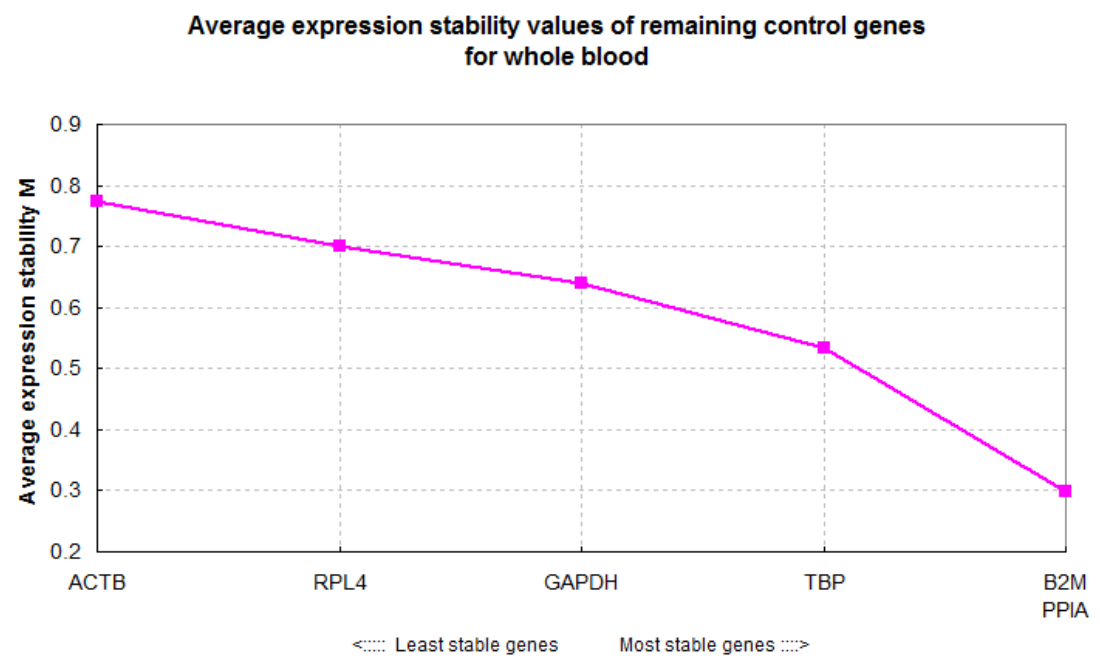

(a)

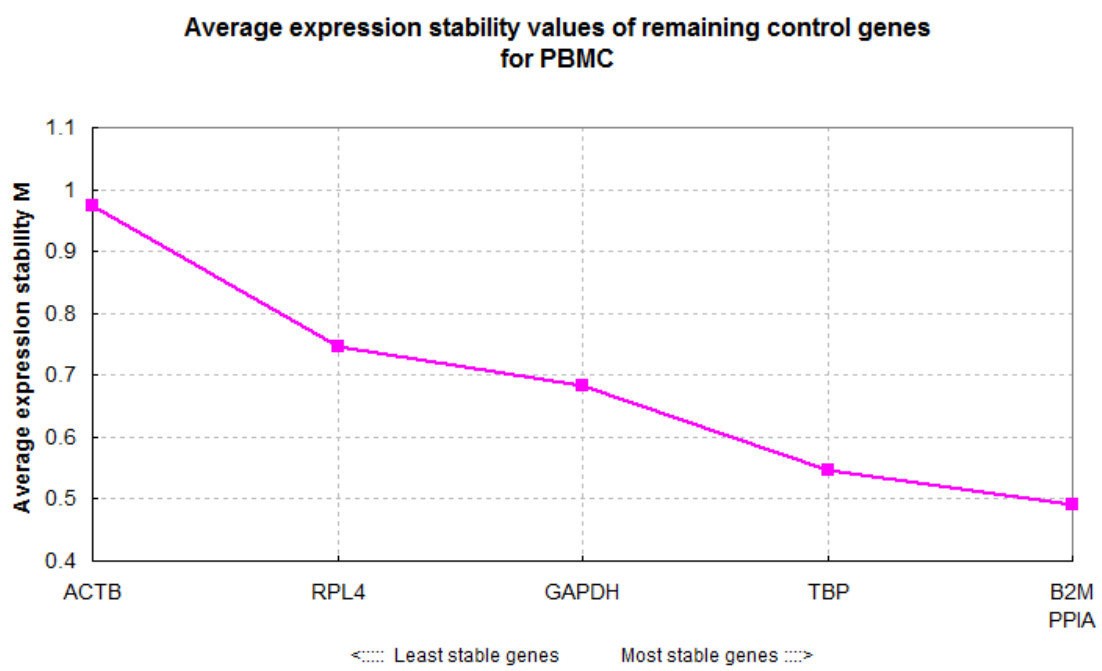

(b)

Figure 2. Average expression stability values $(\mathrm{M})$ of remaining control genes during stepwise exclusion of the least stable control gene (a) whole blood; (b) peripheral blood mononuclear cells (PBMC).

We observed the lowest variation $\mathrm{V}_{\mathrm{n}} / \mathrm{V}_{\mathrm{n}+1}$ between inclusion of the fourth and fifth most stable reference gene $(0.136$ and 0.149 for the whole blood and PBMC).

\section{Gene expression stability assessment using NormFinder}

The gene expression stability was also analyzed by another analysis program, NormFinder, where stable gene expression is indicated by lower average expression stability values. According to NormFinder results as shown in Figure 4, the six candidate genes were more stably expressed in whole blood than PBMC, and also had a similar trend of expression stability in the sample of whole blood and PBMC. Especially, TBP was ranked as the most stable reference gene in both whole blood and PBMC, while $B 2 M$ and PPIA were the second and third most stable ones. NormFinder found the stability value for best combination of two genes, TBP and PPIA, were 0.636 and 1.623 for whole blood and PBMC respectively. In accordance with results valuated by geNorm, the frequently used reference genes $A C T B$ and GAPDH exhibited substantial variation, and were among the three least stable genes.

\section{DISSUSSION}

Whole blood and PBMC, consisting of many types of immune cells are important in vitro models to investigate the immune response to stimulation by environment or pathogens. As an analogue of viral double-stranded RNA, poly I:C is widely used as immunologic stimulant to study the immune regulation of an organism to the RNA viruses.

Previous studies have indicated that different reference 


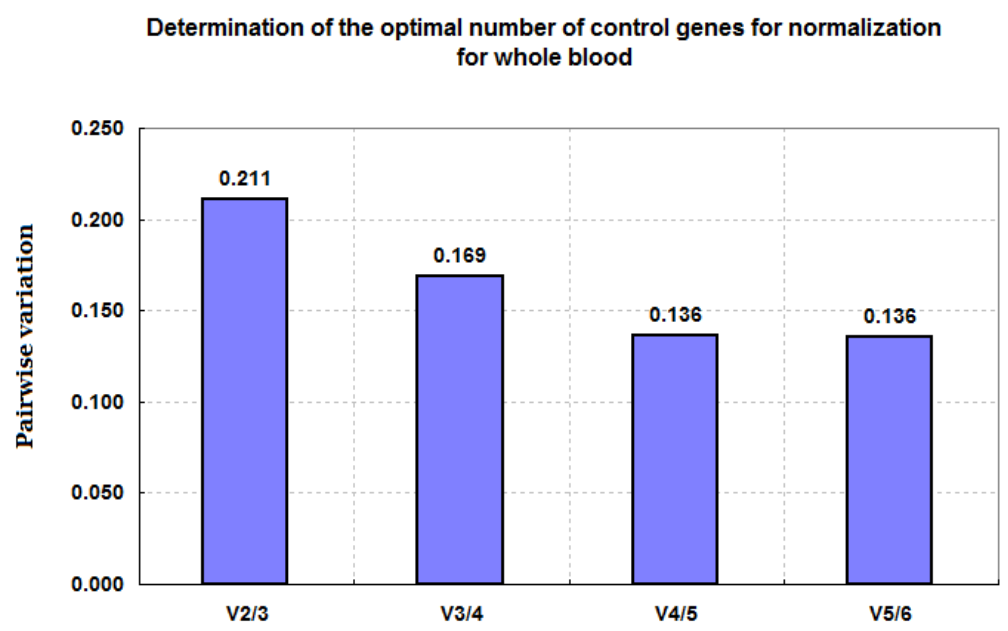

(a)

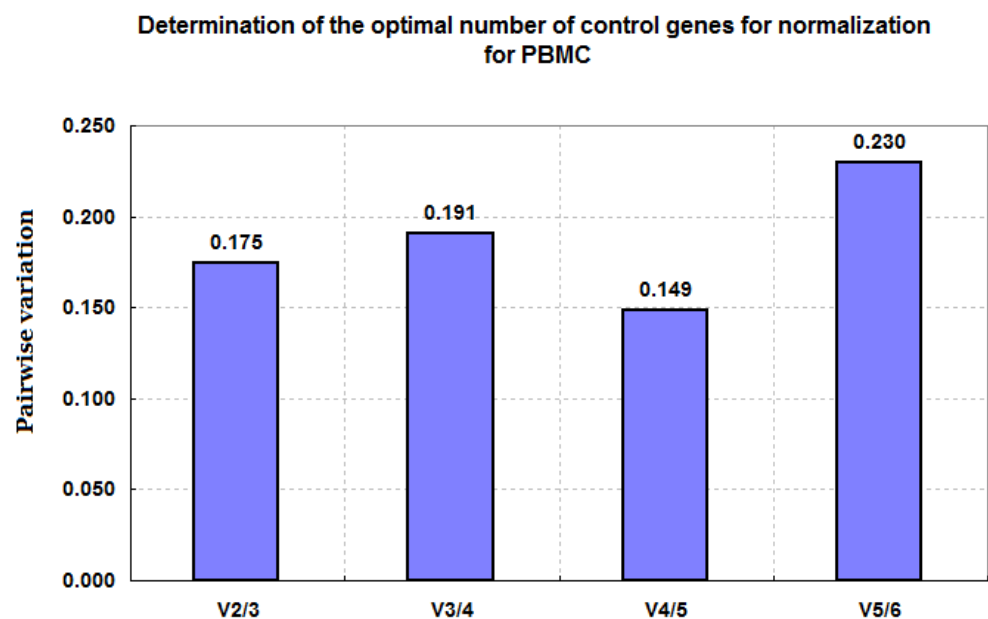

(b)

Figure 3. Pairwise variation $\left(\mathrm{V}_{\mathrm{n}} / \mathrm{V}_{\mathrm{n}+1}\right)$ analysis between the normalization factors $\mathrm{NF}_{\mathrm{n}}$ and $\mathrm{NF}_{\mathrm{n}+1}$. (a) whole blood; (b) peripheral blood mononuclear cells (PBMC).

genes were required for gene expression studies in PBMC at different times or with different immunologic stimulants. For instance, RPL4 and TBP ranked as the two most stably expressed genes in PBMC of pigs at day 1, day 7, day 14 after heat stress, while $B 2 M$ was the most stable at day 21 after heat stress (Xiang-Hong et al., 2011); PPIA, B2M, and RPL4 were the most stable reference genes in Lipopolysaccharide (LPS) stimulated PBMC, while Tyrosine 3-monooxygenase/tryptophan 5-monooxygenase activation protein, zeta polypeptide (YWHAZ), RPL4 and PPIA were the most stably expressed reference genes in the case of lipoteichoicacid (LTA) stimulated PBMC (Cinar et al., 2012; Cinar et al., 2013). So far, no studies were performed to validate the stability of reference genes in cultured porcine whole blood and PBMC in response to poly I:C stimulation.

There are a number of programs that allow assessment of multiple reference genes, such as geNorm (Vandesompele et al., 2002), NormFinder (Andersen et al., 2004) and Bestkeeper (Pfaffl et al., 2004). But to date, there is no consensus on which method should be used to examine reference gene expression stability. In particular, Radoni et al. (2005) suggested that ranking lists of the genes tested was tool-dependent. A comparison of different algorithms of reference gene selection allows a better evaluation of the most reliable controls and reduces the risk of artificial selection of co-regulated transcripts. Therefore, in the present study, we used two statistical approaches, geNorm and NormFinder, to evaluate the gene expression stability of the six reference genes, and also compare the results obtained using these two programs.

Based on the geNorm programs, the most stable reference genes in both whole blood and PBMC were PPIA and $B 2 M$. Combination of the two genes can obtain rather 


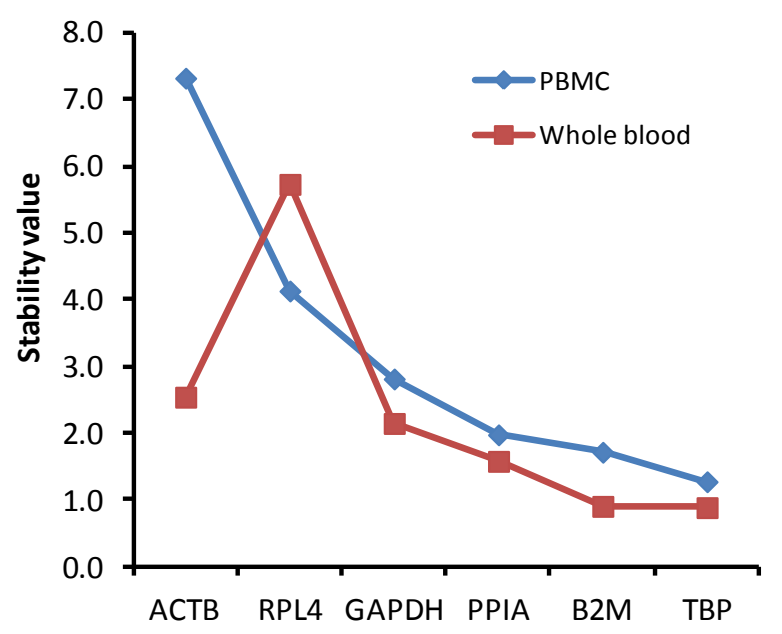

Figure 4. Gene expression stability of the six candidate reference genes calculated by NormFinder.

small $\mathrm{M}$ values for the samples of whole blood (0.299) and PBMC (0.490). As experimental data from the geNorm developers have shown that stably expressed genes typically exhibit mean $\mathrm{M}$ values lower than 0.5 in relatively homogeneous sample panels (Hellemans et al., 2007). Thus, the combining of the two genes, PPIA and B2M, is appropriate for normalization of gene expression of whole blood and PBMC. On the other hand, based on the NormFinder, TBP was ranked as the most stable reference gene in both whole blood and PBMC, while genes $B 2 M$ and PPIA were the second and third ones. This discrepancy is probably due to the different algorithms used in the two programs. Though the most stable genes were different, the first three most stable genes were the same as evaluated by the two programs.

It has been suggested that using the geometric mean of two or more reference genes for qRT-PCR studies might generate more reliable results (Vandesompele et al., 2002). In order to determine the optimal number of required reference genes, we also performed pairwise variation $\left(\mathrm{V}_{\mathrm{n}} / \mathrm{V}_{\mathrm{n}+1}\right)$ analysis by geNorm, as shown in Figure 3. Based on the cut-off value of 0.15 proposed by geNorm program, four reference genes are necessary to obtain the lowest control genes in the tested samples of both whole blood and PBMC. In practice, it is not always possible to measure multiple reference genes due to limited sample availability and cost. Additionally, the cut-off value of 0.15 is an empirical value and should not be taken as a too strict cut off value, as it is already suggested by the geNorm manual itself. Therefore, we recommend using the geometric mean of the first three stable genes, TBP, PPIA, and $B 2 M$, to normalize the gene expression of whole blood and PBMC with poly I:C stimulation.

Up to now, most of the published qRT-PCR results were normalized to either GAPDH or ACTB without experimentally confirming their invariant expression under the experimental conditions described. In our present analyses, these two genes were the least and the third least stable genes among the six candidates. Consistent with our result, some previous studies also reported $A C T B$ and $G A P D H$ were unsuitable as reference genes in whole blood (Brym et al., 2013) and PBMC (Spalenza et al., 2011; Xiang-Hong et al., 2011) and other specific samples (Uddin et al., 2011; Zhang et al., 2012). ACTB is one of the major components of cytoplasmic microfilaments in eukaryotic cells and plays an important role in diverse cellular functions, while GAPDH not only acts as a component of the glycolytic pathway but also takes part in other processes as well. Both $A C T B$ and $G A P D H$ have sophisticated functions in organisms, and their expression profile might fluctuate according to the corresponding experimental conditions. Our analysis results confirmed, once more, blindly choosing reference genes is no longer acceptable, and authors must accurately validate candidate reference genes before use in gene expression qRT-PCR studies.

\section{ACKNOWLEDGEMENTS}

The work reported in this paper was supported by grant from the National Natural Science Foundations of China (31201779), the Agricultural Improved Variety Engineer of Shandong Province (2011LZ013-01, 2011LZ013-02) and Shandong swine industry technology system.

\section{CONFLICT OF INTEREST STATEMENT}

We certify that there is no conflict of interest with any financial organization regarding the material discussed in the manuscript.

\section{REFERENCES}

Andersen, C. L., J. L. Jensen, and T. F. Ørntoft. 2004. Normalization of real-time quantitative reverse transcriptionPCR data: a model-based variance estimation approach to identify genes suited for normalization, applied to bladder and colon cancer data sets. Cancer Res. 64:5245-5250.

Brym, P., A. Ruść, and S. Kamiński. 2013. Evaluation of reference genes for qRT-PCR gene expression studies in whole blood samples from healthy and leukemia-virus infected cattle. Vet. Immunol. Immunopathol. 153:302-307.

Cao, Y., Z. Lu, Y. Li, P. Sun, D. Li, P. Li, X. Bai, Y. Fu, H. Bao, and C. Zhou. 2013. Poly (I: C) combined with multi-epitope protein vaccine completely protects against virulent foot-andmouth disease virus challenge in pigs. Antiviral Res. 97:145153.

Caskey, M., F. Lefebvre, A. Filali-Mouhim, M. J. Cameron, J. P. Goulet, E. K. Haddad, G. Breton, C. Trumpfheller, S. Pollak, I. Shimeliovich, A. Duque-Alarcon, L. Pan, A. Nelkenbaum, A. 
M. Salazar, S. J. Schlesinger, R. M. Steinman, and R. P. Sékaly. 2011. Synthetic double-stranded RNA induces innate immune responses similar to a live viral vaccine in humans. J. Exp. Med. 208:2357-2366.

Cinar, M. U., M. A. Islam, M. Pröll, H. Kocamis, E. Tholen, D. Tesfaye, C. Looft, K. Schellander, and M. J. Uddin. 2013. Evaluation of suitable reference genes for gene expression studies in porcine PBMCs in response to LPS and LTA. BMC Res. Notes 6:56.

Cinar, M. U., M. A. Islam, M. J. Uddin, E. Tholen, D. Tesfaye, C. Looft, and K. Schellander. 2012. Evaluation of suitable reference genes for gene expression studies in porcine alveolar macrophages in response to LPS and LTA. BMC Res. Notes 5:107.

Facci, M. R., G. Auray, F. Meurens, R. Buchanan, J. van Kessel, and V. Gerdts. 2011. Stability of expression of reference genes in porcine peripheral blood mononuclear and dendritic cells Vet. Immunol. Immunopathol. 141:11-15.

Gao, Y., L. Flori, J. Lecardonnel, D. Esquerré , Z. L. Hu, A. Teillaud, G. Lemonnier, F. Lefèvre, I. Oswald, and C. RogelGaillard. 2010. Transcriptome analysis of porcine PBMCs after in vitro stimulation by LPS or PMA/ionomycin using an expression array targeting the pig immune response. BMC Genomics 11:292.

Hellemans, J., G. Mortier, A. De Paepe, F. Speleman, and J. Vandesompele. 2007. qBase relative quantification framework and software for management and automated analysis of realtime quantitative PCR data. Genome Biol. 8:R19.

Huggett, J., K. Dheda, S. Bustin, and A. Zumla. 2005. Real-time RT-PCR normalisation; strategies and considerations. Genes Immun. 6:279-284

Martino, A., M. Cabiati, M. Campan, T. Prescimone, D. Minocci, C. Caselli, A. M. Rossi, D. Giannessi, and S. Del Ry. 2011. Selection of reference genes for normalization of real-time PCR data in minipig heart failure model and evaluation of TNF- $\alpha$ mRNA expression. J. Biotechnol. 153:92-99.

McCartney, S., W. Vermi, S. Gilfillan, M. Cella, T. L. Murphy, R. D. Schreiber, K. M. Murphy, and M. Colonna. 2009. Distinct and complementary functions of MDA5 and TLR3 in poly (I: C)-mediated activation of mouse NK cells. J. Exp. Med. 206:2967-2976.

Meurens, F., A. Summerfield, H. Nauwynck, L. Saif, and V. Gerdts. 2012. The pig: a model for human infectious diseases. Trends Microbiol. 20:50-57.
Nygard, A. B., C. B. Jørgensen, S. Cirera, and M. Fredholm. 2007. Selection of reference genes for gene expression studies in pig tissues using SYBR green qPCR. BMC Mol. Biol. 8:67.

Pfaffl, M. W., A. Tichopad, C. Prgomet, and T. P. Neuvians. 2004. Determination of stable housekeeping genes, differentially regulated target genes and sample integrity: BestKeeperExcel-based tool using pair-wise correlations. Biotechnol. Lett. 26:509-515.

Radonić, A., S. Thulke, H.-G. Bae, M. A. Müller, W. Siegert, and A. Nitsche. 2005. Reference gene selection for quantitative real-time PCR analysis in virus infected cells: SARS corona virus, Yellow fever virus, Human Herpesvirus-6, Camelpox virus and Cytomegalovirus infections. Virol. J. 2:7.

Spalenza, V., F. Girolami, C. Bevilacqua, F. Riondato, R. Rasero, C. Nebbia, P. Sacchi, and P. Martin. 2011. Identification of internal control genes for quantitative expression analysis by real-time PCR in bovine peripheral lymphocytes. Vet. J. 189:278-283.

Uddin, M., M. Cinar, D. Tesfaye, C. Looft, E. Tholen, and K. Schellander. 2011. Age-related changes in relative expression stability of commonly used housekeeping genes in selected porcine tissues. BMC Res. Notes 4:441.

Uddin, M. J., P. K. Nuro-Gyina, M. A. Islam, D. Tesfaye, E. Tholen, C. Looft, K. Schellander, and M. U. Cinar. 2012. Expression dynamics of Toll-like receptors mRNA and cytokines in porcine peripheral blood mononuclear cells stimulated by bacterial lipopolysaccharide. Vet. Immunol. Immunopathol. 147:211-222.

Vandesompele, J., K. De Preter, F. Pattyn, B. Poppe, N. Van Roy, A. De Paepe, and F. Speleman. 2002. Accurate normalization of real-time quantitative RT-PCR data by geometric averaging of multiple internal control genes. Genome Biol. 3:research0034- research0034.11.

Xiang-Hong, J., Y. Yan-Hong, X. Han-Jin, A. Li-long, X. YingMei, J. Pei-Rong, and L. Ming. 2011. Selection of reference genes for gene expression studies in PBMC from Bama miniature pig under heat stress. Vet. Immunol. Immunopathol. 144:160-166

Zhang, J., Z. Tang, N. Wang, L. Long, and K. Li. 2012. Evaluating a set of reference genes for expression normalization in multiple tissues and skeletal muscle at different development stages in pigs using quantitative real-time polymerase chain reaction. DNA Cell Biol. 31:106-113. 